\title{
Alternative Cellular Energy (ACE) pathway activation as the mode of action of neutral red dye phototherapy of human viruses
}

\begin{abstract}
Ultraviolet (UV) light illumination of herpes simplex virus (HSV) skin lesions pretreated with freshly prepared neutral red dye leads to near immediate symptomatic relief and expedites the subsequent healing of the lesions. This therapy is similarly effective in the recovery from both shingles caused by herpes zoster virus (HZV) and warts caused by human papillomavirus (HPV). The present paper describes a series of findings consistent with the hypothesis thathealing occurs via activation of the alternative cellular energy (ACE) pathway. Of particular note is the appearance of UV skin and hair fluorescence beyond the treated lesions and the healing that can simultaneously occur in both directly treated and more distant untreated skin lesions. Neutral red induced fluorescence of intact skin occurring in the sites of previous HSV outbreaks may offera surrogate marker for HSV shedding and/or impending recurrence and providesthe opportunity for preventative phototherapy. Systemic non-immunological activation of the ACE pathway also holdspromise in the therapy of other virus infections for which the immune response is inadequate.
\end{abstract}

Keywords: Herpes virus, Warts, Zoster, Shingles, Papillomavirus, Post-herpetic neuralgia, Phototherapy, Alternative cellular energy, ACE pathway, ACE pigments, KELEA
Volume I Issue 4 - 2014

\author{
W John Martin,' Jon Stoneburner' \\ 'Institute of Progressive Medicine, USA \\ 2Private Practice, Bradenton, USA
}

Correspondence: W. John Martin, Medical Director, Institute of Progressive Medicine, 1634 Spruce Street, South Pasadena CA 91030, USA, Tel 626-616-2868, Email wjohnmartin@ccid.org

Received: August 26, 2014 | Published: September 16, 2014
Abbreviations: HSV, Herpes Simplex Virus; HZV, Herpes Zoster Virus; HPV, Human Papillomavirus; ACE, Alternative Cellular Energy; KELEA, Kinetic Energy Limiting Electrostatic Attraction; UV, Ultraviolet; CPE, Cytopathic Effect

\section{Introduction}

The discovery that certain dyes could stain various microbes dates to the late nineteenth century. Dr. Paul Ehrlich was among the first to suggest that dyes might be useful as antimicrobial agents. ${ }^{1}$ The microbe-killing capacity of certain dyes was markedly enhanced with light. $^{2}$ and this was later shown to commonly involve the formation of reactive oxygen molecules. ${ }^{3}$ This oxygen-requiring indirect killing mechanism was referred to as photodynamic therapy in contrast to phototherapy, which did not involve the formation of toxic free radicals. ${ }^{4,5}$ Both approaches to the suppression of bacterial infections were largely discarded as antibiotics became available, although a triple dye therapy is still used to prevent infection of the umbilical cords of approximately half of all births. ${ }^{6}$

Since antibiotics were not active in treating virus infections, consideration was given to the possible use of dyes plus light as antivirus agents. ${ }^{7}$ Particular emphasis was placed on neutral red dye since it was regularly being used to monitor virus cultures. ${ }^{8}$ The dye was mainly employed to distinguish between dead cells, which did not stain and surrounding living cells, which did stain. Some investigators noted, however, an increase in neutral red staining of early-infected cells compared to the lower level of live staining of uninfected cells. ${ }^{9}$ Moreover, the addition of neutral red dye to virus inocula reduced infectivity when the cultures were subsequently maintained in a lighted environment. The neutral red dye was presumed to directly interact with virus particles leading to their photodynamic destruction. ${ }^{10-12}$

The successful clinical use of neutral red dye plus light treatment of HSV skin lesions was originally reported in 1971 by Felber. ${ }^{13}$ along with colleagues, he published additional findings in $1973 .{ }^{14}$ Although, many clinics began utilizing this approach, the results were seemingly too variable to be accepted into mainstream medicine. The issue also arose as to whether neutral red dye plus light could mutate the virus DNA in such a manner that the damaged virus could potentially cause cancer. ${ }^{15-17}$ In a major clinical trial published in 1975 in the New England Journal of Medicine, Dr. Meyer's and his colleagues from Harvard Medical School reported that neutral red dye applied to HSV skin lesions followed by light illumination was ineffective. ${ }^{18}$ Additional publications supported this negative conclusion and the neutral red dye/light procedure was largely abandoned.

Also in the early 1970's, Stoneburner observed the destruction of neutral red stained HSV infected cells in cultures when viewed under UV light. This observation led him to use a UV light source in the neutral red therapy of HSV infected skin lesions. He further extended the basic approach to treating shingles caused by HZV and genital warts caused by HPV. Collaboration was established between the two authors of this paper to pursue the underlying science of the healing mechanism.

\section{Materials and methods}

Bulk quantities of neutral red dye (CAS number 553-24-2) are available from Dudley Chemicals, Lakewood, New Jersey, which is the primary supplier of this product to other chemical companies within the United States. UV-A lights in the form of 2 or 4 foot-long fluorescent BLB tubes and condensed spiral UV bulbs are widely available through hardware and lighting stores, such as Feit Electric (Pico Rivera, CA) and Halco (Norcross, GA). A Wood's lamp, commonly used in dermatology offices is also a suitable source of UV lighting.

Direct treatment of active HSV and HZV skin lesions: As described previously, ${ }^{20}$ the lesions are gently debrided using a moistened paper towel or sponge to open existing vesicles or to remove the crusting from older lesions. Neutral red dye is dissolved into $10 \mathrm{ml}$ of tap water 
at approximately $0.5 \mathrm{mg} / \mathrm{ml}$, although concentrations ranging from 0.1 to $1.0 \mathrm{mg} / \mathrm{ml}$ are quite satisfactory. Three drops of Chlorox $(8.25 \%$ sodium chlorite) are added to create a slightly alkaline solution, but still retain the red color of the dye. Using a Q-tip, the dye is applied to the debrided skin lesion. UV illumination is provided at a distance of approximately 6 inches, which is sufficiently close to evoke fluorescence from the treated lesions. Illumination times generally range from 30 to 60 minutes.

Indirect treatment of active HSV skin lesions: Rather than directly applying the neutral red dye to the lesion, it is applied via a spray to the portion of the moistened paper towel used to debride the lesion. Prior to spraying the neutral red dye, the paper towel is laid onto the herpes skin lesion, which can be first covered with a layer of Saran $\square$ wrap to exclude physical contact of the dye with the herpes lesion. The paper towel is then UV illuminated and the fluorescing area, containing debrided material from the lesion, is placed directly over the underlying skin lesion. After several minutes, the paper towel can be removed and UV illumination more directly applied to the now fluorescing skin lesion and continued for 30-60 minutes.

Treatment of skin areas of prior genital HSV outbreaks: The patient is advised not to bath or shower for 24 hours. A moistened paper towel is used to collect material from the skin areas on which prior outbreaks have occurred. As in the indirect treatment of active lesions, the towel is laid onto the skin, with or without an intervening layer of Saran $\square$ wrap, and sprayed with a freshly prepared dilute solution $(\sim 0.1 \mathrm{mg} / \mathrm{ml})$ of neutral red dye. The underlying skin is periodically assessed for UV fluorescence and when seen, direct illumination of the skin is continued for as long as the fluorescence is clearly visible up to an hour. If bright fluorescence is still persisting, the patient is instructed to return the next day for a repeated similar treatment. For some patients, the neutral red dye is directly sprayed onto the skin to test for the presence of fluorescing materials and if detected the UV illumination is continued till the fluorescence fades.

Treatment of post-herpetic neuralgia: An inquiry is made of the patient to broadly indicate the painful region. The skin over this area is then either swabbed or sprayed with dilute neutral red dye and examined for any noticeable fluorescence. If fluorescence is not seen, a 26-gauge needle is used to create very fine superficial puncture wounds into the skin at approximately 1" intervals in a checkerboard pattern. The needle probing is just sufficient to elicit minute spots of visible blood, which mix with the neutral red dye previously applied to the skin. Upon UV illumination, at least one focus of fluorescence will generally be observed. If not, the probing can be extended even beyond the symptomatic area. When a focus is identified, a series of 10 or so additional skin punctures are made within an approximate 1" circular area to essentially create a patch of tissue-derived fluorescing material. This area is then lightly sprayed with additional dilute neutral red dye and UV illumination continued for up to an hour As the UV illumination of this area is continued, the surrounding skin will typically also begin to fluorescence in a slowly expanding manner. Illumination is continued till either the fluorescence fades or an hour has elapsed.

Treatment of warts: A minute quantity of sterile neutral red dye solution is injected into the center of one or more of the presenting warts. The injection is made by applying slight pressure to the plunger of a tuberculum syringe fitted with a 26 gauge needle. The injected warts will typically show discernable, reddish-purple UV fluorescence. UV illumination of the injected wart(s) is continued for up to an hour. Note, when many warts are present, only a few of the warts need to be injected for the procedure to be continued into the illumination phase.

\section{Results}

The primary purpose of this paper is to describe various observations, which may help lead to the concise understanding of the healing mechanism of using neutral red dye and UV light in the therapy of HSV, HZV and HPV skin lesions.

Direct therapy of active HSV oral and genital lesions: Figure 1 is a composite of 4 photos taken of a male patient undergoing neutral red dye/UV therapy of a HSV lesion of the upper lip. The upper left photo shows the herpes lesion prior to debridement and application of neutral red dye. The stained lesion fluoresced upon UV illumination as did an area near the junction of the upper and lower lips. The photograph is mainly intended to show the intense yellow fluorescence seen at 5 minutes into the phototherapy (upper right photo) and how the fluorescence gradually fades, being significantly less at 15 minutes (lower left photo). The lower right photograph shows the healing, asymptomatic lesion at 8 hours after therapy.

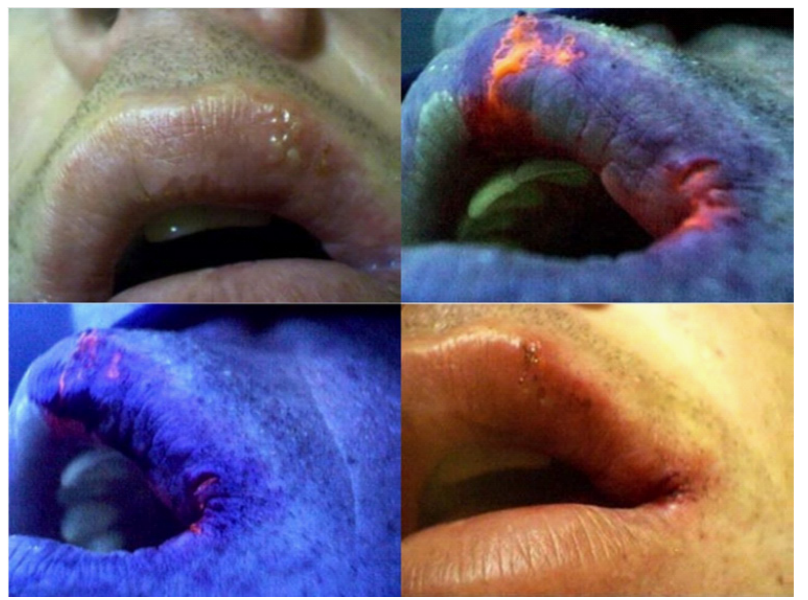

Figure I Neutral red dye phototherapy of a HSV infected lip. The upper left photo shows the lesion prior to its debridement and the application of neutral red dye to both the left upper and lower lips. The upper right photo shows the striking fluorescence seen in the area of the main herpes lesion, as well as fluorescence occurring in lesions at the corner of the mouth. This photo was taken at 5 minutes into the therapy. The intensity of the fluorescence in both areas of the lip gradually faded with continued UV illumination. This is shown in the lower left photo taken at 15 minutes into the therapy. A lower right photo is of the treated lip 8 hours after the therapy. It shows the expedited healing process, which is typically near completion by 24 hours after the neutral red dye phototherapy.

The fluorescence of active HSV lesions requires the presence of neutral red dye and may not occur immediately after applying the neutral red dye. Figure 2 left upper photograph shows a herpes lip lesion prior to therapy. The upper right photograph was taken 10 seconds after the application of the neutral red dye to the lip. It shows no fluorescence, although fluorescence is clearly present at 5 minutes (lower left photo). The treatment led to subsequent healing of the lesion as seen in the lower right photograph taken 24 hours after treatment. Patients will sometimes report a slight tingling sensation within the lesion during the early phase of the therapy as well as the relief of any pain if previously present.

Figure 3 shows the pre-therapy appearance of an inflamed early penile herpes outbreak (left upper photo). The upper right photo was taken shortly after gentle debridement and application of neutral red dye to the entire penis, followed by UV illumination. This photo clearly shows two prominent foci of bright fluorescence; which gradually faded over a 30-minute period. The lesions also became asymptomatic during this period. The lower left photo was obtained 
24 hours after therapy, at which time only slight residual scarring was present without any signs of inflammation. The lower right photo of this composite Figure is a further example of neutral red induced fluorescence of genital herpes seen during phototherapy. As will be reported elsewhere, skin lesions that fluoresce with neutral red dye are not necessarily herpes. Still, it is of potential diagnostic interest that several genital skin lesions, which did not fluoresce upon the application of neutral red dye, were later shown by serology to be not due to herpes infection.

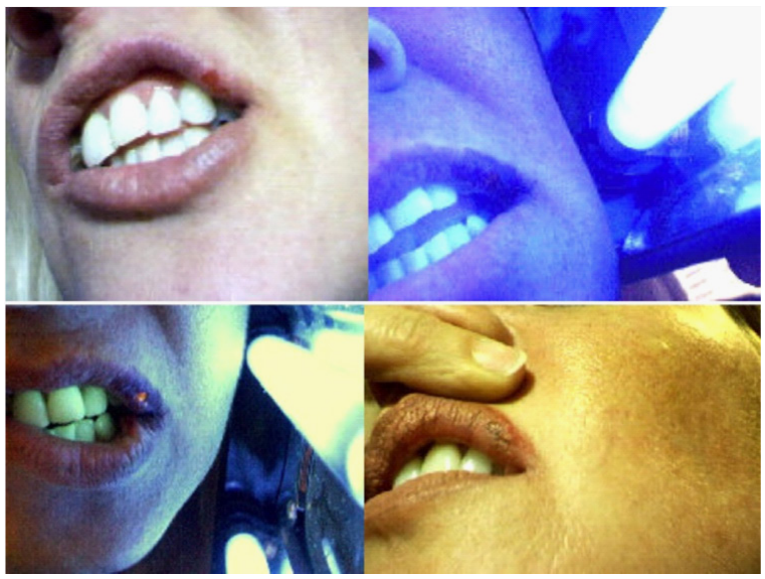

Figure 2 Neutral red dye phototherapy of another HSV infected lip. The Figure is intended to show that fluorescence of herpes lesions is often not immediately apparent with the application of the neutral red dye, but requires some seconds to develop and further time to increase in intensity. The upper left photo shows the lesion prior to therapy. The upper right photo was taken 10 seconds after the neutral red dye was applied and UV illumination commenced. No fluorescence was apparent at this time point. The lesion showed bright fluorescence at 5 minutes into therapy (lower left photo). The lower right photo shows the largely healed, slightly scabbed lesion as seen 24 hours after therapy.
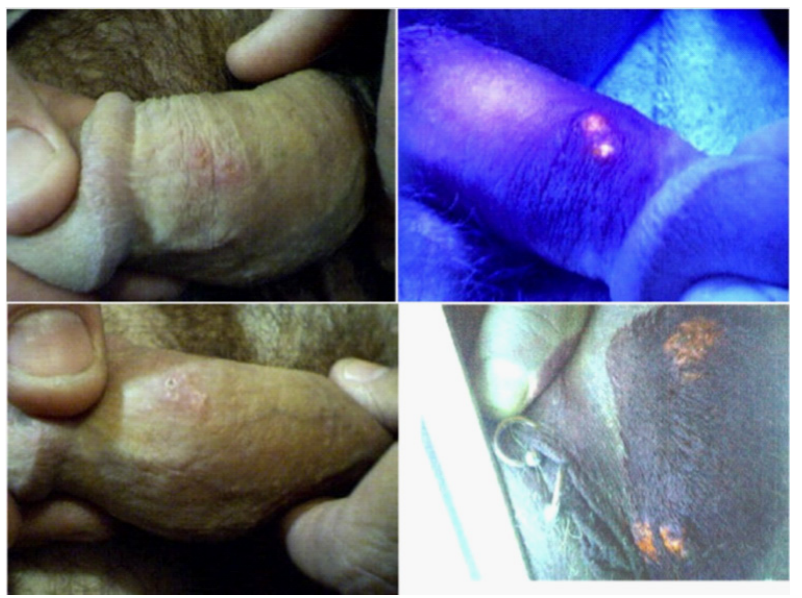

Figure 3The left upper photo shows an early HSV outbreak on the penis. Neutral red dye was applied to the penis. Upon UV illumination, the affected area showed two foci of easily discernable fluorescence (upper right photo). Even with continuing UV illumination, the fluorescence of the two foci subsequently faded (not shown). The lower left photo is of the largely healed penile lesion a day after treatment The figure also includes a photograph of UV fluorescence seen in several regions of the labia of a HSV infected patient after the application of neutral red dye. The lesions were far more conspicuous using neutral red dye with UV illumination, than upon direct observation of the labia.

Figure 4 is a photograph of multiple HZV skin lesion in the buttock of a female patient. The photographs show the lesions i) prior to treatment (upper left); ii) following debridement and application of neutral red dye (upper right);iii) evoked fluorescence following UV illumination (lower left) and iv) appearance 7 days following therapy (lower right). The lower left photograph is particularly noteworthy because it also shows considerable fluorescence, not only of the main lesion, but also on the skin to the right side and lower down from this lesion. Note also that the fluorescence of HZV lesions tends to be a duller, yellow-orange color, in contrast to the brighter yellow fluorescence elicited from acute HSV lesions. The photograph also shows a droplet, which formed due to the oozing of fluorescing fluid from the lesion. Considerable swelling of herpes lesions undergoing dye/light therapy is commonly observed from the ingress of tissue fluid. While clear under visible light, the leaked fluids readily fluoresceupon UV illumination and can be collected for further examination

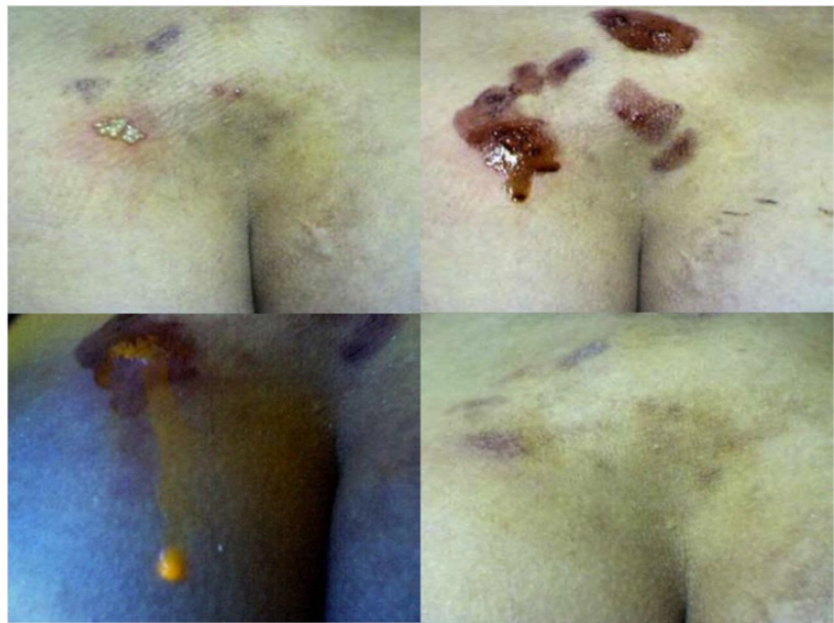

Figure 4 This Figure shows a series of before, during and after therapy photographs of lesions occurring in the buttock. The lesions were clinically identified as being shingles, although they had periodically recurred in the patient. They are shown before therapy in the upper left photo. Neutral red dye was applied after the lesions were debrided (upper right photo). The lower left photograph is included to mainly show the marked swelling of treated lesions, which can occur during therapy. The swelling is mainly the result of the ingress of tissue fluid, which in this case, actually formed into a large droplet draining from the lesion. This photograph also shows the yellow-orange fluorescence of the lesion and of the fluid coming from the lesion. The fluid coming out of the lesion was clear when seen under regular lighting. The lower right photo shows the healing achieved using the neutral red dye phototherapy.

The upper left photo in Figure 5 shows a relatively large penile wart, with several additional smaller warts. The large wart was injected with neutral red dye and UV illumination begun. The reddish purple fluorescence of the injected wart is apparent in the upper right photograph. At this early stage, the other warts did not fluoresce, but gradually did so during the next 15 minutes. Over this period, the injected wart progressively increased in size to almost that of a small grape. It then dramatically burst, releasing fluorescing material onto much of the penis. The UV illumination was discontinued and the remaining lesion following its eruption photograph and shown in the lower left photograph. During the period of UV illumination, a slight UV fluorescent urethral discharge was also noted (lower right photo). All of the warts subsequently underwent expedited healing.

Figure 6 left photograph illustrates neutral red induced fluorescence of pubic hairs in a patient with genital warts. A small quantity of neutral red dye was sprayed onto the pubic area above the penis. Not only did the sprayed hairs fluoresce, but within several minutes hairs elsewhere on the perineum also fluoresced (right photograph). 
Fluorescence of pubic hairs and within uninvolved areas of skin is not uncommonly observed in patients undergoing therapy of nearby genital herpes lesions. No direct fluorescence of the skin and/or pubic hair is normally seen until neutral red dye is applied to the lesions and/ or directly to the skin or hairs.

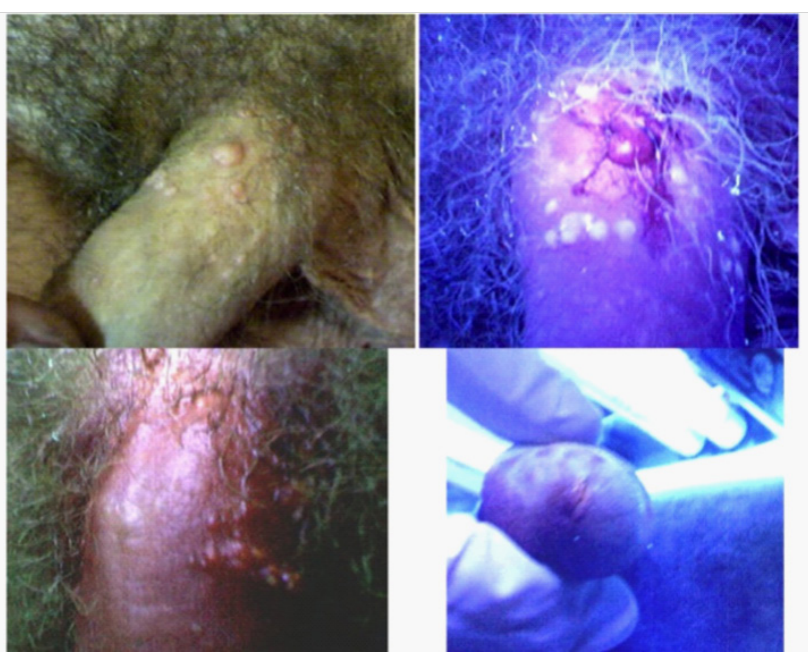

Figure 5 Neutral red dye phototherapy of genital warts. The patient had a major genital wart on his penis, along with many smaller nearby warts (upper left photo). A small amount of neutral red dye was injected into the larger wart with some spillage occurring onto the surrounding skin as the injection needle was withdrawn. The penis was then UV illuminated. The upper right photo shows the reddish-purplish fluorescence occurring shortly, thereafter, in the injected wart. The nearby warts appear white and at this early time point but gradually also fluoresced. Moreover, a slight urethral discharge of fluorescing fluid was discernable during the therapy (lower left photograph). As the UV illumination continued, the injected wart greatly increased in size to that of a grape before abruptly bursting and releasing fluorescing material onto the penis. The lower right photograph was taken under bright light illumination at the end of the period of UV illumination. It shows the minimal, residual tissue from the burst wart. There was complete healing of the penis within several days of the therapy.

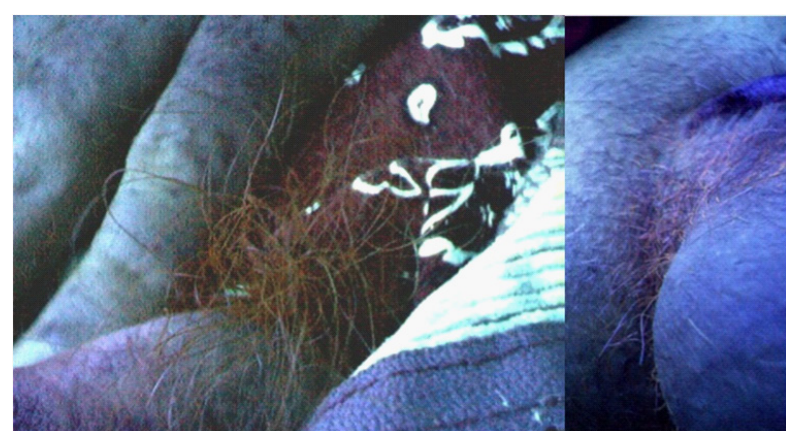

Figure 6 This Figure shows reddish fluorescence on pubic hairs at the base of the penis of a patient prior to therapy of a genital wart. A small quantity of dilute neutral red dye was sprayed onto the pubic hair and the area examined for fluorescence. Not only did the sprayed hairs fluoresce (left photo), but so too did unsprayed hairs situated elsewhere on the perineum (right photo).

Materials that fluoresce when stained with dilute neutral red dye, can occasionally also be collected in the absence of any active lesion. A wet sponge or paper towel is used to rub the skin and collect dried perspiration, primarily from the area of the prior herpes outbreak. If UV illumination of the collected material is arranged so that it occurs in close vicinity of the skin area from which the material was collected; the underlying skin will usually begin to show direct UV inducible fluorescence. This observation is basically similar to that described earlier in which neutral red dye stained material collected from an active herpes skin lesion is used to induce direct UV fluorescence of the lesion with subsequent healing. The procedure involving the fluorescing of material collected from skin areas of prior herpes outbreaks has, accordingly, provided an ovel means of treating patients in whom no active lesion is currently present. Based on anecdotal reports from several patients, this procedure has greatly reduced the likelihood of future recurrences.

Rather than attempting to collect materials, a dilute neutral red dye spray can be used to directly test for the presence of fluorescing materials on the skin and/or hair in patients without any active skin lesions at the time of examination. On some occasions, a particularly bright, fluorescing focus is observed on essentially intact skin and may foretell a site of active virus shedding and/or of an impending clinical herpes lesion. Although, very occasionally reported. ${ }^{21,22}$ we have never noted an allergic reaction to neutral red dye applied to the skin.

For patients with post-herpetic neuralgia, neural red dye fluorescing material is usually not easily detected on the skin prior to therapy. Instead, it has been customary to draw fluorescing material onto the skin by first locating a tissue area containing the material and making multiple superficial needle-punctures in this area. Upon UV illumination, the area of skin fluorescence will gradually spread over several inches. More impressively, patients will commonly report relief of neuralgic pain within minutes of beginning the therapy. Moreover, the relief is sustained for long periods, with only an occasional patient requiring repeated treatments, and these times because of shingles, rather than the recurrence of pain. Well over one hundred post-neuralgic symptomatic patients have benefited from this therapy.

Figure 7 is a striking example of the regression achieved in a patient with a large condyloma acuminatum (HPV papillomatous) lesion of the tongue. The lesion was injected at multiple sites with a total of approximately $0.2 \mathrm{ml}$ of neutral red dye. The upper left photograph is of the lesion taken shortly after the injections. An additional few particles of neutral red dye powder were sprinkled onto the lesion just prior to the lesion being illuminated for 1 hour with an external UV light, positioned close to the open mouth. Significant necrosis of the lesion developed over the next several hours. The upper right photograph was taken at 3 hours after the UV illumination and shows the height of the purulent discharge coming from the lesion, which had an unpleasant smell. The acute reaction resolved so that by day 3 the gradually shrinking lesion was essentially non-inflammatory. By 3 weeks, the normal contours of the tongue had largely been restored with the exception of a small posterior papilloma (lower left photograph). There was also palpable, residual firmness within the affected side of the tongue. A second therapy was, therefore, given, with the patient subsequently reporting complete resolution. Prior to therapy, the patient was finding it very difficult to consume anything other than liquids. This was because of his tongue lesion as well as radiologically detected papillomatous lesions of the esophagus and larynx. Within days of receiving treatment, the patient regained his ability to consume solid food. Furthermore, the patient coughed up several pieces of non-inflamed, dead tissue, as the tongue lesion was regressing. An example of a necrotic but not overtly inflamed piece of tissue cough by the patient and collected onto a Q-tip is shown in the lower right photograph (Figure 7).

The healing of distant lesions has been observed in other patients. For example, Figure 8 shows the before-therapy presence and the post-therapy regression of warts on the dorsum of the foot of a patient undergoing successful dye/light therapy of multiple penile genital 
warts (Figure 9). Other examples include the regression of oral herpes lesions upon treating of genital herpes and the regression of a herpes lesion involving the thumb upon treating a lip lesion. Inflammation is not a noticeable feature of the regression occurring in either the directly treated or the non-treated distant lesions.

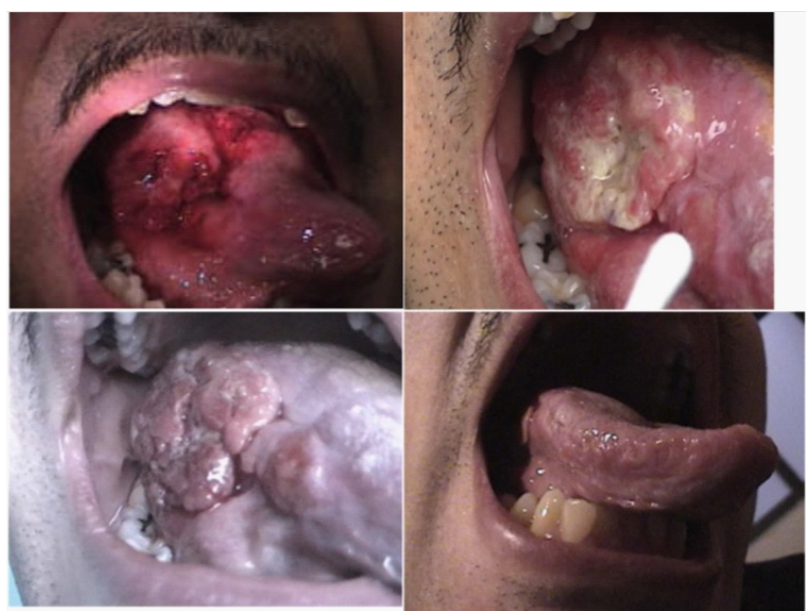

Figure 7 The upper left photograph shows the condyloma acuminatum growth of the tongue shortly after it was injected with neutral red dye, with some additional dye particles placed onto the surface of the lesion. UV illumination was applied for an hour within the hospital setting. Necrosis accompanied by a purulent discharge rapidly developed and a photograph taken 3 hours after the end of the UV illumination is shown in the upper right photograph. This acute reaction subsided and the lesion progressively decreased in size. A photograph taken at 3 days and a photograph taken a t 3 weeks are respectively shown in the lower left and lower right panels of this figure. A repeat treatment was given at 3 weeks because of some remaining palpable thickness in the lateral tongue. This treatment proceeded without the swelling seen with the first two treatments.

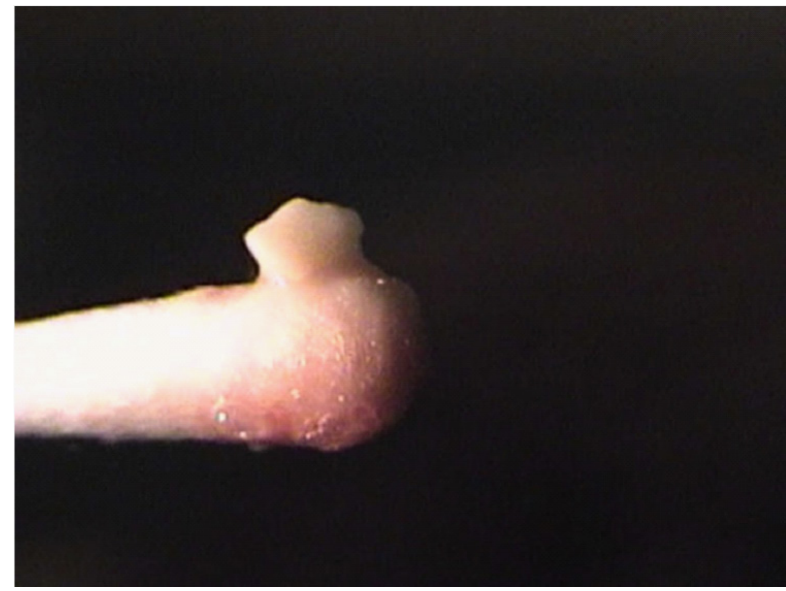

Figure 8 One of numerous pieces of necrotic, but seemingly non-inflamed tissues expelled by coughing during the time that the tongue lesion was regressing.

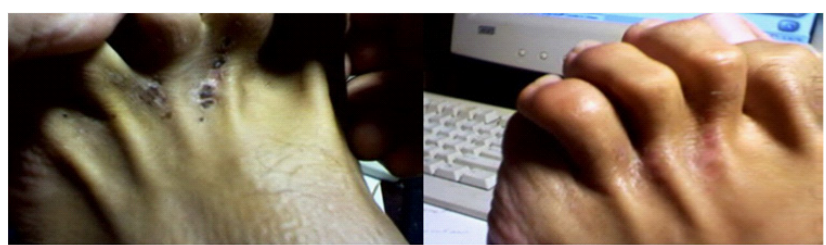

Figure 9 This Figure shows the regression of warts on the dorsum of the foot of a patient treated for multiple penile warts. The foot photograph was taken 24 hours after the treatment was applied to the genital warts, which also underwent regression.

\section{Discussion}

This paper presents an important new medical paradigm within the expanding field of energy-based medicine. It proposes a nonimmunological, anti-virus defense mechanism involving the body's production of natural materials. These materials fluoresce under UV illumination, especially upon the addition of certain dyes including neutral red. At least partial insight into the nature of these fluorescing materials has come from in vivo and in vitro studies on stealth adapted viruses. ${ }^{23-35}$ These viruses are not effectively recognized by the cellular immune system, but can, nevertheless, be suppressed in infected patients and in virus-inoculated animals. ${ }^{25,26}$ Suppression can also be seen in virus cultures. ${ }^{30}$ The in vitro suppression is mediated by a proposed ACE pathway, which utilizes materials termed ACE pigments. Specifically, cultures of stealth adapted viruses undergo a striking cellular repair of the cytopathic effect (CPE) as cell-derived ACE pigment particles accumulate in infrequently re-fed cultures. The particles will fluoresce and show other energy transducing (converting) activities; with the fluorescence being enhanced using neutral red, acridine orange and other dyes. Rapid reactivation of the CPE occurs in the cultures by simple replacement ACE pigmentcontaining tissue culture medium with fresh tissue culture medium. Reactivation does not occur, however, if particles from the media of repaired cultures are first added to the fresh re-feeding medium. ${ }^{30}$ Similar, intra- and extra-cellular particles have been identified in brain biopsies of stealth adapted virus infected individuals. ${ }^{31}$ as well as on hair and in dried perspiration from stealth adapted virus infected patients..$^{32}$ Interestingly, the mitochondria in many of the brain cells containing complex particles (inclusions) were seen to be severely disrupted, leading to the suggestions that the particles were providing an alternate (non-mitochondria) source of cellular energy. ${ }^{31}$ The ACE pathway is envisioned as a source of cellular energy distinguishable from the cellular energy derived from food calories. More recent studies have shown a link between the ACE pathway and a kinetic property of the body's fluids, which can also be displayed in water into which ACE pigments or related materials are added. It has been proposed that by way of separated electrical charges, dipolar ACE pigments absorb a natural environmental energy force tentatively called KELEA (kinetic energy limiting electrostatic attraction). Possibly via an oscillatory mechanism, energized ACE pigments are able to transfer kinetic energy to the body's fluids.$^{33}$

The ACE pathway concept challenges two commonly held tenets regarding virus infections. These are: i) That the immune system is the sole natural mechanism by which humans and animals can eliminate established virus infections and ii) that the antimicrobial activity of various dyes, with or without the use of light, is necessarily due to the direct killing of the microbes.

With regards to the first tenet, recurrent HSV infections and even periodic shedding of HSV have near universally been equated with lapses of immune defense. Reports of stress induced herpes relapses have accordingly been interpreted as evidence for a psychoneuroimmunology network, with immune failure being ultimately responsible for the outbreak. ${ }^{34}$ A proposed direct interaction of the neural system with the ACE pathway ${ }^{33}$ provides a more satisfactory interpretation of this association; especially since stress activation of herpes infections is even observed in corals. ${ }^{35}$ which do not have an established immune system.

Similarly, the role of the neutral red dye in conjunction with UV light in the therapy of herpes infections can now be proposed as primarily a means of enhancing the body's ACE pathway. It does so in a manner in which even distant ACE pigments will more readily 
fluoresce upon direct illumination with UV light. The activation of ACE pigments elsewhere in the body seemingly occurs without the necessity of the distant ACE pigments being directly UV illuminated. Moreover, the ACE pigment-activating signal is not simply fluorescence, since as shown in other studies, black plastic sheeting can be used in place of Saran $\square$ wrap, to separate fluorescing ACE pigments collected onto paper towels from the underlying skin being treated. In essence, a biophysical systemic activation of the ACE pathway is seemingly being achieved by the localized use of neutral red dye plus UV illumination as an initial triggering mechanism.

The systemic nature of the healing response can also explain why many of the HSV patients treated using the present method have reported becoming free of further recurrences. Indeed several patients have even reported on becoming herpes antibody negative. This would be an important finding since it may well reverse the axiom that "herpes is forever."

The rather remarkable cessation of post-herpetic neuralgia following localized therapy is also suggestive of a systemic effect. It is also of special interest that treatment can also be provided even without the need for an active lesion. The method described in this paper, still requires the collection of ACE pigments from the patient's skin. This need can potentially be replaced with the use of natural materials with ACE pigment-like activities, and even more easily with the use of activated solutions with added neutral red dye. This latter approach has already been used with several HSV infected patients without active lesions. It will be of interest to follow the serological status of patients participating in these studies.

As will also be reported elsewhere, these developments have opened up the use of ACE pathway activation to the potential therapy of many additional types of virus infections A major application of the method has been to the suppression of infections caused by stealth adapted viruses. ${ }^{33}$ Another important potential target for therapy is HIV infections, in which the immune system is rendered ineffective. Comparative clinical studies are needed to assess the relative ease and effectiveness of applying different methods of ACE pathway activation to infectious and even to non-infectious illnesses.

\section{Conclusion}

An explanation is offered for the healing of HSV, HZV and HPV skin lesions using UV illumination in conjunction with freshly prepared neutral red dye. Rather than photodynamic destruction of viruses, the procedure more likely leads to the systemic activation of the alternative cellular energy (ACE) pathway. The materials rendered UV fluorescent with neutral red dye are referred to as ACE pigments and appear to be produced as part of a major non-immunological defense mechanism. The experience gained in using the dye/light therapy in herpes and papillomavirus infections may well lead to future major applications of ACE pathway activation to other illnesses. Even as presently used, ACE pathway activation offers many clinical benefits, especially if HSV outbreaks and shedding can be prevented and the agony of shingles and post-shingles neuralgia eliminated.

\section{Acknowledgment}

The work was sponsored by MI Hope Inc. a non-profit public charity.

\section{References}

1. Kasten FH Paul Ehrlich: pathfinder in cell biology. 1. Chronicle of his life and accomplishments in immunology, cancer research, and chemotherapy. Biotech Histochem. 1996;71(1):2-37.
2. Raabe $\mathrm{O}$ Uber die wirking fluoreszierender stoffe auf infusorien. Zeitschrift Biol. 1990;39:524-526.

3. Müller-Breitkreutz K, Mohr H, Briviba K et al. Inactivation of viruses by chemically and photochemically generated singlet molecular oxygen. $J$ Photochem Photobiol B. 1995;30(1):63-70.

4. Ledoux-Lebards C Action de la lumiSre sur la toxicitg de l'eosin et de quelques autres substances pour les paramecies. Annal Institut Pasteur. 1902;16:593.

5. Von Tappeiner H, Jodlbauer A Uber die Wirkung der photodynamischen (fluoreszier-enden) Stoffe auf Infusorien. Dtsch Arch Klin Med. 1904;80:427-487

6. Suliman AK, Watts $\mathrm{H}$, Beiler $\mathrm{J}$ et al. Triple dye plus rubbing alcohol versus triple dye alone for umbilical cord care. Clin Pediatr (Phila). 2010;49(1):45-48.

7. Wallis C, Melnick JL Photodynamic inactivation of animal viruses: a review. Photochem Photobiol 1965;4(2):159-170.

8. Dulbecco R, Vogt M Plaque formation and isolation of pure lines with poliomyelitis viruses. J Exp Med. 1954;99(2):167-182.

9. Schmidt NJ, Dennis J, Lennette EH Plaque reduction neutralization test for human cytomegalovirus based upon enhanced uptake of neutral red by virus-infected cells. J Clin Microbiol. 1976;4(1):61-66.

10. Crowther D, Melnick JL The incorporation of neutral red and acridine orange into developing poliovirus particles making them photosensitive. Virology. 1961;14:11-21.

11. Tomita Y, Prince AM Photodynamic inactivation of arborviruses by neutral red and visible light. Proc Soc Exptl Biol Med. 1963;112(4):887-890.

12. Wallis C, Scheiris C, Melnick JL Photodynamically inactivated vaccines prepared by growing viruses in cells containing neutral red. J Immunol. 1967;99(6):1134-1139.

13. Felber TD Presentation to Scientific Assembly of AMA Section on Dermatology, June 21, 1971; Reported as "Photoinactivation may find use against herpesvirus". JAMA. 1971;217:270.

14. Felber TD, Smith EB, Knox JM et al. Photodynamic inactivation of herpes simplex: report of a clinical trial. JAMA. 1973;223(3):289-292.

15. Rapp F, Li JL, Jerkofsky M Transformation of mammalian cells by DNA-containing viruses following photodynamic inactivation. Virology. 1973;55(2):339-346.

16. Bockstahler LE, Lytle CD, Hellman KB A review of photodynamic therapy for herpes simplex: benefits and potential risks. DHEW Publication (FDA) 1974; No. 75-8013. Reprinted in N Y J Dent. 1974;45:148-157.

17. Li JL, Jerkofsky MA, Rapp F Demonstration of oncogenic potential of mammalian cells transformed by DNA-containing viruses following photodynamic inactivation. Int J Cancer. 1975;15(2):190-202.

18. Myers MG, Oxman MN, Clark JE et al. Failure of neutral-red photodynamic inactivation in recurrent herpes simplex virus infections. $N$ Engl J Med. 1975;293(19):945-949.

19. McKinlay JB, Zeikus JG Extracellular iron reduction is mediated in part by neutral red and hydrogenase in Escherichia coli. Appl Environ Microbiol. 2004;70(6):3467-3474.

20. Martin WJ, Stoneburner J Symptomatic relief of herpetic skin lesions utilizing an energy-based approach to healing. Exp Mol Pathol. 2005;78(2):131-134.

21. Mitchell JC, Stewart WD Allergic contact dermatitis from neutral red applied for herpes simplex. Arch Dermatol. 1973;108(5):689.

22. Goldenberg RL, Nelson K Dermatitis from neutral red therapy of herpes genitalis. Obstet Gynecol 1975;46(3):359-360. 
23. Martin WJ, Zeng LC, Ahmed K, Roy M Cytomegalovirus related sequences in an atypical cytopathic virus repeatedly isolated from a patien with the chronic fatigue syndrome. Am J Pathol. 1994;145(2):440-451.

24. Martin WJ, Ahmed KN, Zeng LC et al. African green monkey origin of the atypical cytopathic 'stealth virus' isolated from a patient with chronic fatigue syndrome. Clin Diagn Virol. 1995;4(1):93-103.

25. Martin WJ, Glass RT Acute encephalopathy induced in cats with a stealth virus isolated from a patient with chronic fatigue syndrome. Pathobiology. 1995;63(3):115-118.

26. Martin WJ Severe stealth virus encephalopathy following chronic fatigue syndrome like illness: Clinical and histopathological features. Pathobiology. 1996;64(1):1-8.

27. Martin WJ Simian cytomegalovirus related stealth virus isolated from the cerebrospinal fluid of a patient with bipolar psychosis and acute encephalopathy. Pathobiology. 1996;64(2):64-66.

28. Martin WJ, Anderson D Stealth virus epidemic in the Mohave Valley. I. Initial report of viral isolation. Pathobiology. 1997;65(1):51-56.

29. Martin WJ, Anderson D Stealth virus epidemic in the Mohave Valley: Severe vacuolating encephalopathy in a child presenting with a behavioral disorder. Exp Mol Pathol. 1999;66(1):19-30.
30. Martin WJ Stealth virus culture pigments: a potential source of cellular energy. Exp Mol Pathol. 2003;74(3):210-223.

31. Martin WJ Complex intracellular inclusions in the brain of a child with a stealth virus encephalopathy. Exp Mol Pathol. 2003;74(3):197-209.

32. Martin WJ Alternative cellular energy pigments mistaken for parasitic skin infestations. Exp Mol Pathol. 2005;78(3):212-214.

33. Martin WJ KELEA activated water - Enhancing the alternative cellular energy (ACE) pathway in "Stealth Adapted Viruses; Alternative Cellular Energy (ACE) \& KELEAÔ Activated Water.” J Prog Med (Volume 1) Author House, Bloomington, IN, USA, pp. 2014;115-144.

34. Irwin MR Human psychoneuroimmunology: 20 years of discovery. Brain Behav Immun. 2008;22(2):129-139.

35. Vega Thurber RL, Barott KL, Hall D et al. Metagenomic analysis indicates that stressors induce production of herpes-like viruses in the coral Porites compressa. Proc Natl Acad Sci U S A. 2008;105(47):18413-18418. 